\title{
Análisis biomecánico de la influencia del calzado deportivo en los esfuerzos presentes en la extremidad inferior
}

\author{
pags 71-81 \\ Grupo de Investigación: Diseño avanzado \\ Línea de investigación: Biomecánica \\ Iván Sánchez•, Juan Díaz»•, \& Héctor Castro»••
}

Recibido: 6 de mayo de 2015

Aceptado: 29 de septiembre de 2015

\section{RESUMEN}

En la actualidad se han realizado numerosas investigaciones con el fin de conocer el comportamiento mecánico de la extremidad inferior la cual es la encargada de la locomoción, durante actividades físicas cotidianas y de alto rendimiento. Sin embargo es difícil encontrar estudios sobre los esfuerzos que se presentan en las articulaciones de miembro inferior con el uso de calzado deportivo convencional durante el trote. El objetivo de este artículo es realizar un análisis biomecánico de la influencia del calzado deportivo en los esfuerzos presentes en la extremidad inferior durante la fase de apoyo en el ciclo del trote. Para esto, se realizó una descripción de la distribución de los esfuerzos mecánicos en las articulaciones del miembro inferior, en presencia y ausencia de la plantilla intermedia para calzado deportivo, por medio de un análisis por elementos finitos. Posteriormente se obtuvieron los esfuerzos de Von Mises para las condiciones de trote descalzo y trote con uso de la plantilla del calzado deportivo. Las simulaciones demostraron que cuando se compara el trote con y sin uso de calzado deportivo, los puntos máximos y mínimos de esfuerzos disminuyen en las articulaciones. En conclusión se encontró que el uso de una plantilla durante el trote presenta un efecto positivo al reducir los fuerzas sobre las articulaciones del miembro inferior.

Palabras clave: Biomecánica, Ciclo de trote, Calzado deportivo, Extremidad inferior

\begin{abstract}
At present the science community have been made researches in order to know the mechanical behavior of the lower limb, which is responsible for the locomotion during daily and high-performance physical activities. However it is difficult to find researches about stresses that arise in the lower limb joints with the use of sports shoes. The aim of this paper is to analyze the biomechanical behavior of the stresses in the lower extremity under influence of sports shoes during the support phase in jogging. Through of finite element method a description of mechanical stresses distributions on lower-limb joints was achieved (with presence and absence of midsole-footwear). Afterward the distribution of Von-Misses stresses was obtained for the barefoot jogging, and jogging with midsole-footwear. The simulations showed that the localization of points with maximum and minimum stresses in the joints are different when the person is jogging without use of sports shoes compared with jogging using sport shoes; the magnitude of these stresses are reduced too. In conclusion have been found that the use of midsole-footwear during jogging has a positive effect because the loads the lower limb joints a
\end{abstract}

Key Words: Biomechanics, Stress distribution on joints, Sports Shoes, Lower Extremity.

\footnotetext{
- Estudiante coinvestigador programa de Ingeniería mecánica, Fundación Universidad de América. ivan.sanchez@estudiantes.uamerica.edu.co

-• Estudiante coinvestigador programa de Ingeniería Mecánica, Fundación Universidad de América. juan.diaz2@estudiantes.uamerica.edu.co.

•. Ingeniero mecatronico especialista en biomecánica, docente Investigador Fundación Universidad de América. hector.castro@profesores.uamerica.edu.co
} 


\section{INTRODUCCIÓN}

La extremidad inferior humana es la encargada de la locomoción, que es la capacidad para moverse de un sitio al otro. Esta función se lleva a cabo gracias a la intervención en grupo de partes esenciales como los son los huesos, las articulaciones y los músculos. Estas articulaciones permiten que el cuerpo se desplace ya sea caminando, trotando, corriendo, saltando o escalando.

El ciclo de la marcha se divide en fase de apoyo, cuando al menos uno de los pies se encuentra en contacto con el suelo, y fase aérea o de balanceo cuando el pie se encuentra en el aire (Dugan \& Bhat, 2005). En el ciclo de trote, la velocidad del cuerpo tiende a aumentar en comparación con el ciclo de caminata, generando así una etapa de apoyo más corta del $40 \%$ del ciclo y una etapa de balanceo más larga, la cual, realizara el $60 \%$ restante del ciclo (Ajay, Sherman, Reinbolt, \& Delp, 2011).

La fase de apoyo se subdivide en tres etapas (Chai, 2003):

- Contacto inicial: sub-fase de impacto

- Apoyo medio: sub-fase de equilibrio

- Salida o despegue del pie. sub-fase de propulsión.

La marcha humana es una práctica cotidiana, la cual generalmente se realiza con el uso de calzado. Esta indumentaria fue creada con el fin de evitar lesiones y dar comodidad al momento de realizar cualquier actividad. Sin embargo, no todo el calzado cumple con esta función, por lo cual, un calzado diseñado para atletas está hecho para soportar el alto entrenamiento físico a diferencia de un calzado deportivo convencional, el cual, desde su diseño, materiales y construcción es diferente, generando la duda de si en realidad este tipo de calzado cumple con la función de amortiguar los impactos contra el suelo y brindar protección contra lesiones.
Varias investigaciones biomecánicas han descrito el comportamiento del miembro inferior sobre la marcha humana. En (Yousif \& Aziz, 2012) se realizó el análisis biomecánico del fémur humano durante la caminata. En (Meardon \& Derrick, 2014) se analizó el efecto de la manipulación del esfuerzo tibial durante el trote. Por su parte en (Andreaus, Colloca, \& Toscano, 2008) se presentó el análisis por elementos finitos del comportamiento del fémur con y sin el uso de una prótesis. En (Dugan \& Bhat, 2005) se investigó la biomecánica y el análisis del ciclo de trote humano. En (De Wit, De Clercq, \& Aerts, 2000) presentó el análisis biomecánico de la fase de apoyo durante la carrera. En (Novacheck, 1998) se analizó la biomecánica de trotar. Por otra parte en la investigación de (Jin-Rae, Seung-Bum, SungHyun, Seung-Ho, \& Shi-Bok, 2009) se presentó el análisis del impacto del calzado deportivo usando un acople 3D entre el pie y el calzado por el método de los elementos finitos. De igual manera, se han realizado investigaciones donde se ha identificado el comportamiento mecánico de los zapatos. En (O'Leary, Vorpahl, \& Heiderscheit, 2008) se presentó el efecto de las plantillas en las fuerzas de impacto durante la carrera. Cámara (Cámara, 2011) comparó la amortiguación durante la caminata con y sin el uso de botas de montaña.

Sin embargo, no se tiene referencia de investigaciones que permitan conocer los esfuerzos que presentan las articulaciones del miembro inferior humano durante la fase de apoyo del ciclo de trote y los cambios que genera el implementar en la actividad física el calzado deportivo convencional.

En este contexto, el objetivo de este artículo es dilucidar los esfuerzos a los que se someten las articulaciones de la extremidad inferior humana cuando se trota con y sin calzado, con el fin de aportar un avance para las personas interesadas en reducir los esfuerzos generados al practicar una actividad física y así disminuir lesiones que se provoquen en el miembro inferior. 
LIINEA DE INVESTIGACIÓN: BIOMECÁNICA

\section{MATERIALES Y MÉTODOS}

\subsection{Descripción del modelo geométrico}

Para crear el modelo tridimensional de las articulaciones de la extremidad inferior se recopilaron imágenes de una tomografía axial computarizada de miembro inferior. El modelo fue reconstruido en el software InVesalius y editado en el software MeshLab con el fin de generar una superficie uniforme. Este procedimiento se realizó para cada hueso de las articulaciones del miembro inferior por aparte. Posteriormente, en el software Inventor, se modelaron los cartílagos articulares para las articulaciones coxofemoral y tibioastragalina, los cuales tienen como función principal aportar una superficie de articulación suave permitiendo el desplazamiento entre los huesos (Meardon \&
Derrick, 2014) y la distribución eficiente de las cargas (Fitzgerald, Kaufer, \& Malkani, 2002). Del mismo modo, para la articulación tibiofemoral se modelaron los meniscos, los cuales son cartílagos fibrosos semicirculares encargados de la absorción de choques y del mantenimiento del equilibrio (Palastanga, Field, \& Roger, 2000). Finalmente se procedió a ensamblar los componentes óseos junto con los componentes articulares. (Figura 1).

Luego de obtener los modelos geométricos de cada articulación del miembro inferior, estos fueron rotados teniendo en cuenta los movimientos anatómicos que presentan las articulaciones para la sub-fases de la fase de apoyo durante el ciclo del trote. Los ángulos de rotación aplicados para los modelos geométricos se pueden observar en la tabla 1.

Figura1. Ensambles para las articulaciones de la extremidad inferior.

A)

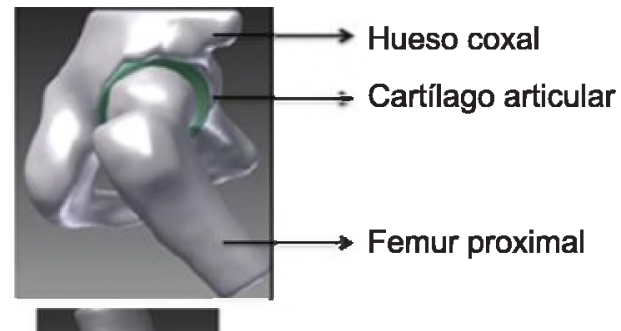

B)

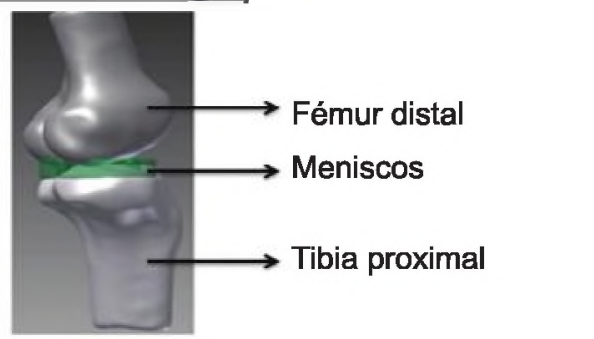

C)

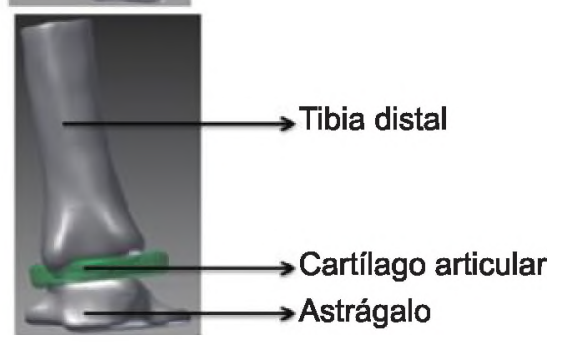

A) Articulación coxofemoral. B) Articulación tibiofemoral. C) Articulación tibioastragalina.

Fuente: los autores 
Tabla 1. Ángulos de rotación de las articulaciones de miembro inferior durante el ciclo de trote.

\begin{tabular}{|c|c|c|c|c|}
\hline & Sub-fase & Ángulo (ㅇ) & Posición & Referencias \\
\hline Articulación & Contacto inicial & 28 & Flexión & \multirow{11}{*}{$\begin{array}{l}\text { (Ajay, Sherman, Reinbolt, \& Delp } \\
\text { 2011) }\end{array}$} \\
\hline \multirow{3}{*}{ coxofemoral } & Apoyo medio & 21 & Flexión & \\
\hline & Salida del pie & -26 & Extensión & \\
\hline & Sub-fase & Ángulo (ㅇ) & Posición & \\
\hline Articulación & Contacto inicial & 13 & Flexión & \\
\hline \multirow[t]{2}{*}{ thbiofemoral } & Apoyo medio & 27 & Flexión & \\
\hline & Salida del pie & 6 & Extensión & \\
\hline \multirow{4}{*}{$\begin{array}{c}\text { Articulación } \\
\text { thbioastragalina }\end{array}$} & Sub-fase & Ángulo (ㅇ) & Posición & \\
\hline & Contacto inicial & 4 & Flexión dorsal & \\
\hline & Apoyo medio & 5 & Flexión plantar & \\
\hline & Salida del pie & 7 & Flexión plantar & \\
\hline
\end{tabular}

Fuente: Los autores

\subsection{Condiciones de cargas y restricciones}

Para este análisis se trabajaron los siguientes músculos: glúteo mayor, aductor mayor, psoas, iliaco, semitendinoso, bíceps femoral, gastrocnemio lateral, gastrocnemio medio, soleo, tibial anterior, tibial posterior extensor de los dedos, peroneo anterior, extensor hallucis. Estos músculos son los encargados de amortiguar e impulsar el cuerpo durante la fase de apoyo del trote (Hammer, Seth, \& Delp, 2010). Para obtener las fuerzas de los músculos se utilizó el programa OpenSim, el cual permite determinar las magnitudes de las fuerzas musculares durante el ciclo del trote, identificando las magnitudes de las fuerzas ejercidas por cada músculo en cada sub-fase de la fase de apoyo del trote (Hammer, Seth, \& Delp, 2010). (Tabla 2).

Para los músculos anteriormente nombrados se obtuvieron las coordenadas del origen " $A$ " y del punto de inserción "B" de cada músculo, lo que permitió la obtención de las componentes rectangulares de las fuerzas en cada sub-fase de la fase de apoyo del trote. Luego, se determinaron las componentes vectoriales de las fuerzas ejercidas por cada músculo.

Para realizar la sumatoria de fuerzas y momentos en la articulación, se definió un punto de corte (PC), el cual se tomó en un segmento del hueso donde este es compacto y está ubicado sobre la diáfisis, en la cual el hueso mantiene su área trasversal tubular o cilíndrica (Universida Veracruzana, 2015). El vector " $\vec{r}$ " une al punto de referencia fijo "PC", con un punto $A$ o $B$, sobre la línea de acción del vector fuerza $\overrightarrow{F m}$. El momento de la fuerza con respecto al punto de corte se define como el producto vectorial de $\vec{r}$ y $\overrightarrow{F m}$.

\subsection{Propiedades mecánicas de los materiales para la simulación}

Para la selección de los materiales usados, en las simulaciones, se recopilaron valores de diferentes autores en donde se especificara cada propiedad mecánica de los huesos, meniscos y cartílagos. Los huesos se componen por dos tipos de materiales compacto y esponjoso. En general el hueso compacto constituye el $80 \%$ del esqueleto, y el esponjoso, el $20 \%$ restante (Tortora \& Reynolds Grabowski, 2012). El tipo de hueso seleccionado para las simulaciones por elementos finitos fue el compacto, debido a que brinda protección y sostén, además de resistir los esfuerzos que se producen con el apoyo y los movimientos producidos en la fase de apoyo del ciclo de trote (Tortora \& Reynolds Grabowski, 2012). Las propiedades de los materiales se definieron como elásticos isotrópicos lineales y se observan en la Tabla 3. 
Sánchez, Iván, et al.: Análisis biomecánico...

LÍNEA DE INVESTIGACIÓN: BIOMECÁNICA

Tabla 2. Músculos de miembro inferior con mayor influencia durante la fase de apoyo del trote.

\begin{tabular}{|l|c|c|c|}
\hline \multicolumn{1}{|c}{ Músculos } & Contacto Inicial & Apoyo Medio & Salida del pie \\
\hline Glúteo mayor 1 & 580,69 & $-576,75$ & $-348,81$ \\
\hline Glúteo mayor 2 & 869,32 & $-816,54$ & $-462,91$ \\
\hline Glúteo mayor 3 & $-609,6$ & $-520,04$ & $-255,44$ \\
\hline Aductor mayor 1 & $-211,35$ & 144,32 & 205,36 \\
\hline Aductor mayor 2 & $-225,4$ & $-177,55$ & 178,06 \\
\hline Aductor mayor 3 & $-486,77$ & $-431,88$ & $-281,68$ \\
\hline Psoas & 866,6 & 926,05 & 961,49 \\
\hline Iliaco & 856,73 & 896,96 & 907,29 \\
\hline Semitendinoso & $-517,3$ & $-494,64$ & $-442,9$ \\
\hline Bíceps femoral cabeza larga & $-1.187,10$ & $-875,61$ & $-682,9$ \\
\hline Gastrocnemio lateral & $-870,48$ & 896,39 & $-671,04$ \\
\hline Gastrocnemio medio & $-1.422,25$ & $1.034,37$ & $-1.528,80$ \\
\hline Tibial anterior & 667,17 & 537,69 & 755,27 \\
\hline Soleo & $1.422,25$ & $1.034,37$ & $-1.810,14$ \\
\hline Extensor de los dedos & 417,73 & 366,97 & 443,01 \\
\hline Tibial posterior & $1.420,25$ & $1.030,37$ & $-1.638,74$ \\
\hline Extensor hallucis & 133,57 & 117,95 & 141,04 \\
\hline Peroneo anterior & 156,24 & 151,07 & 158,01 \\
\hline
\end{tabular}

Fuente: los autores

Tabla 3. Propiedades mecánicas de los materiales para la simulación

\begin{tabular}{|c|c|c|c|}
\hline Elemento & $\begin{array}{l}\text { Modulo de young } \\
\text { (mpa) }\end{array}$ & $\begin{array}{c}\text { Coeficiente de } \\
\text { poisson }\end{array}$ & Referencia \\
\hline Hueso cortical & 10.000 & 0,34 & CHEN, Weng-Ping. (2003) \\
\hline Meniscos & 250 & 0,45 & BEILLAS (2004) \\
\hline Cartílagos & 10 & 0,4 & CHO, Jin-Rae. (2003) \\
\hline
\end{tabular}

Fuente: los autores 


\subsection{Comportamiento del calzado deportivo}

Para analizar el comportamiento del calzado, se trabajó la plantilla de la mitad ya que ésta es la encargada de reducir el impacto al momento de hacer contacto con el suelo (Jin-Rae, Seung-Bum, Sung-Hyun, Seung-Ho, \& Shi-Bok, 2009). Se tomaron cuatro muestras de etilvinilacetato (EVA) de diferente dureza, posteriormente se eligió la muestra que al aplicarle una misma presión, se haya deformado más, la cual simularía la plantilla media de un zapato deportivo y convencional.

Se trabajó con un peso corporal de $65,9 \mathrm{Kg}$, y un área de contacto en la plantilla de $13,75 \mathrm{~cm}^{2}$, lo cual determinó el esfuerzo con el que se comprimieron las muestras, para posteriormente determinar cuál se deformó más, teniendo en cuenta este parámetro se determinó que la muestra cuatro es la más adecuada como se observa en la gráfica1.

Se realizó el cálculo de la energía potencial de deformación, que es la capacidad del material de disipar la energía, por medio de la compresión que tuvo la muestra. Se trabajó como un porcentaje de la energía total generada al impactar el suelo, estableciendo de esta manera, el porcentaje de amortiguación.

Los resultados de amortiguación obtenidos se observan en la Tabla 3.

Grafica 1. Deformación muestras de EVA

Resultados del ensayo

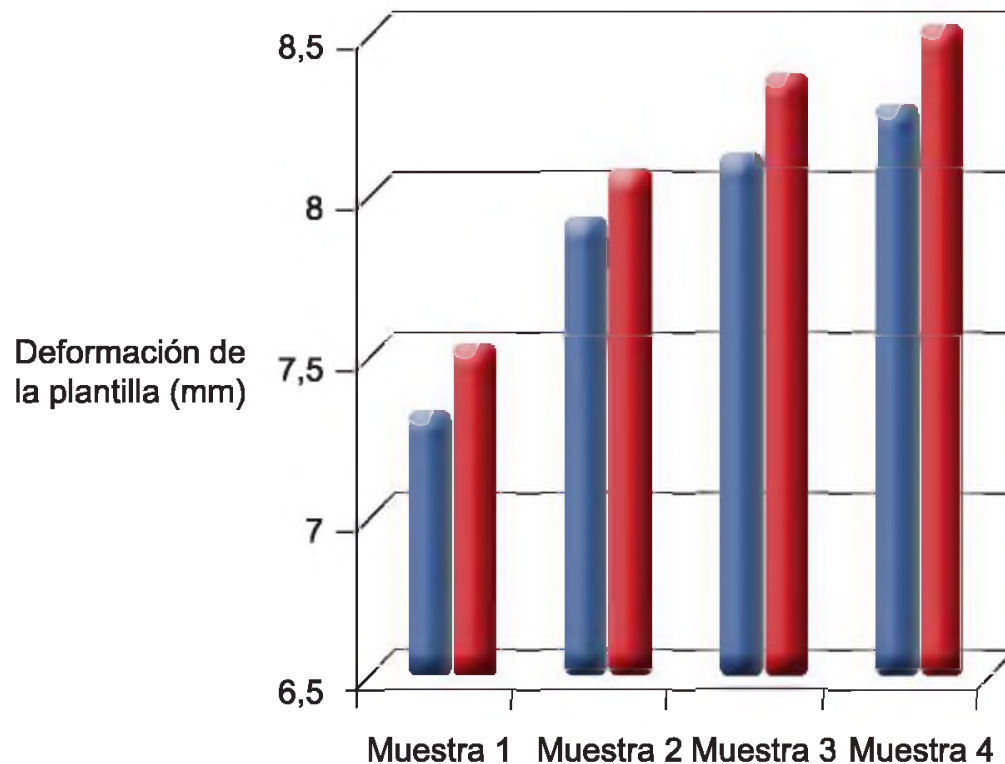

Fuente: los autores
Esfuerzo o presión en el pistón $\sigma p=760,7 \mathrm{psi}\left(10,5 \mathrm{~kg} / \mathrm{cm}^{2}\right)$

Esfuerzo o presión en el pistón $\sigma p=970,7 \mathrm{psi}\left(13,4 \mathrm{~kg} / \mathrm{cm}^{2}\right)$ 
LIINEA DE INVESTIGACIÓN: BIOMECÁNICA

Tabla 3. Resultados amortiguación. (BW) peso corporal, (J) joule.

\begin{tabular}{|c|c|c|c|c|c|c|c|}
\hline \multirow[t]{2}{*}{ Fase } & \multirow{2}{*}{$\begin{array}{c}\text { Peso corporal } \\
\text { (BW) }\end{array}$} & \multicolumn{2}{|c|}{ Energía disipada (0) } & \multicolumn{2}{|c|}{ Amortiguación (\%) } & \multicolumn{2}{|c|}{$\begin{array}{l}\text { Reducción del peso corporal } \\
\text { (BW) }\end{array}$} \\
\hline & & Método1 & Método2 & Método1 & Método2 & Método1 & Mátodo2 \\
\hline $\mathrm{Cl}$ & 2,20 & 11,73 & 9,66 & 9,64 & 7,94 & 1,98 & 2,02 \\
\hline SP & 2,80 & 12,09 & 10,01 & 9,92 & 8,21 & 2,52 & 2,57 \\
\hline
\end{tabular}

Fuente: los autores

\subsection{Restricciones de movimiento al modelo}

Para realizar la simulación por elementos finitos, es necesario aplicar las cargas, momentos y restricciones correspondientes para cada articulación. En la articulación coxofemoral y femorotibial, se aplicó la fuerza en el corte ubicado sobre el fémur, en el caso de la articulación tibioastragalina, las fuerzas fueron aplicadas en el corte ubicado en la tibia, todas la fuerzas son trasladadas al punto de corte, de esta manera determinar los esfuerzos que las mismas generan sobre esta parte del hueso, como se han desarrollado anteriores investigaciones. Por consiguiente, el hueso coxal, la tibia proximal y el astrágalo fueron restringidos en su movimiento. (Tabla 4)

El esfuerzo que se revisó en las simulaciones fue el de Von Mises.

Tabla 4. Cargas y restricciones en las articulaciones del miembro inferior humano

Articulación coxofemoral
Articulación tibioastragalina

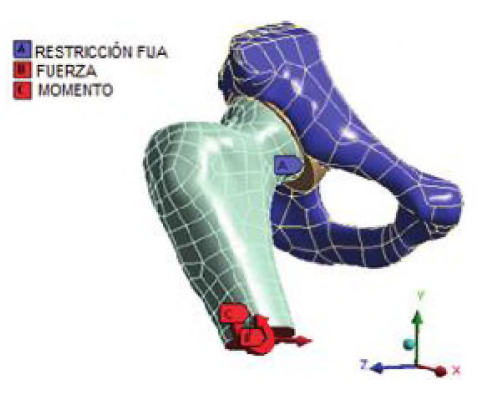

Articulación tibiofemoral

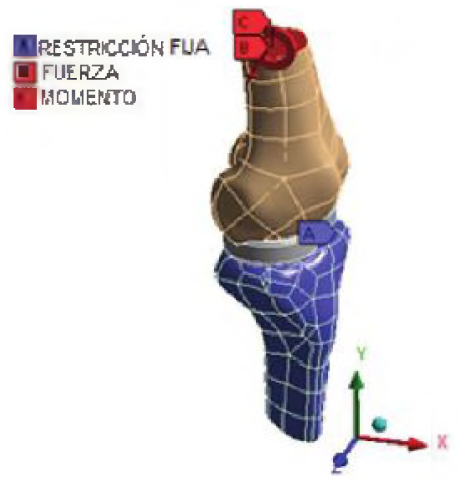

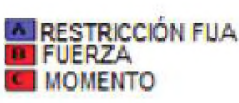

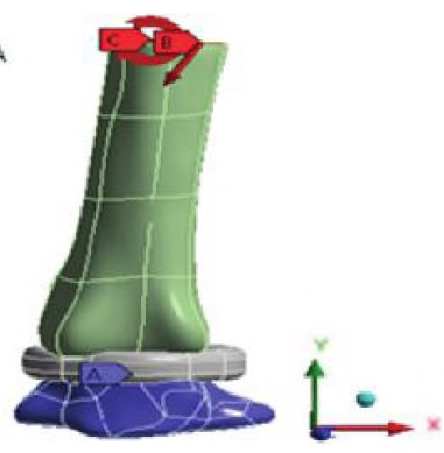

Fuente: los autores 


\section{RESULTADOS}

\subsection{Resultados sin uso de calzado deportivo}

En la simulación para la articulación coxofemoral, el esfuerzo máximo se presentó en el cuello femoral con un valor de $44,63 \mathrm{MPa}$ para la fase de contacto inicial. El cartílago articular presentó un esfuerzo máximo de 5,48 $\mathrm{MPa}$. Se observa que para la articulación coxofemoral el esfuerzo va disminuyendo a medida que se cambia de fase.

Para la articulación tibiofemoral, el esfuerzo máximo se encuentra ubicado en la diáfisis del fémur con un valor de 44,92 Mpa para la fase apoyo medio. Los meniscos evidenciaron un esfuerzo máximo de $37,51 \mathrm{MPa}$ debido a que son los encargados de amortiguar y equilibrar la articulación tibiofemoral.

En el caso de la articulación tibioastragalina, el esfuerzo máximo se evidencio en la parte pos- terior de la tibia (Meardon \& Derrick, 2014) con un valor de 67,22 Mpa para la fase de salida del pie. El cartílago articular presento un esfuerzo máximo de 5,11 MPa. La articulación tibioastragalina presenta un incremento de valores de esfuerzo a medida que se cambia de fase. Los resultados de la simulación se puede observar en la gráfica 2 .

\subsection{Resultados con el uso de calzado deportivo}

El esfuerzo máximo en la articulación coxofemoral con el uso de calzado deportivo fue de $40,17 \mathrm{MPa}$ en la fase de contacto inicial, el cual se generó sobre el cuello femoral. El cartílago articular mostro un esfuerzo máximo de 4,93 MPa.

Para la articulación tibiofemoral, el esfuerzo máximo con el uso de calzado deportivo fue de 44,90 MPa ubicado en el fémur distal. Los meniscos presentaron un esfuerzo máximo de 37 , $5 \mathrm{MPa}$. En ambos caso el máximo esfuerzo se presentó en la fase de apoyo medio.

Gráfica 2. Resultados obtenidos sin uso de calzado deportivo

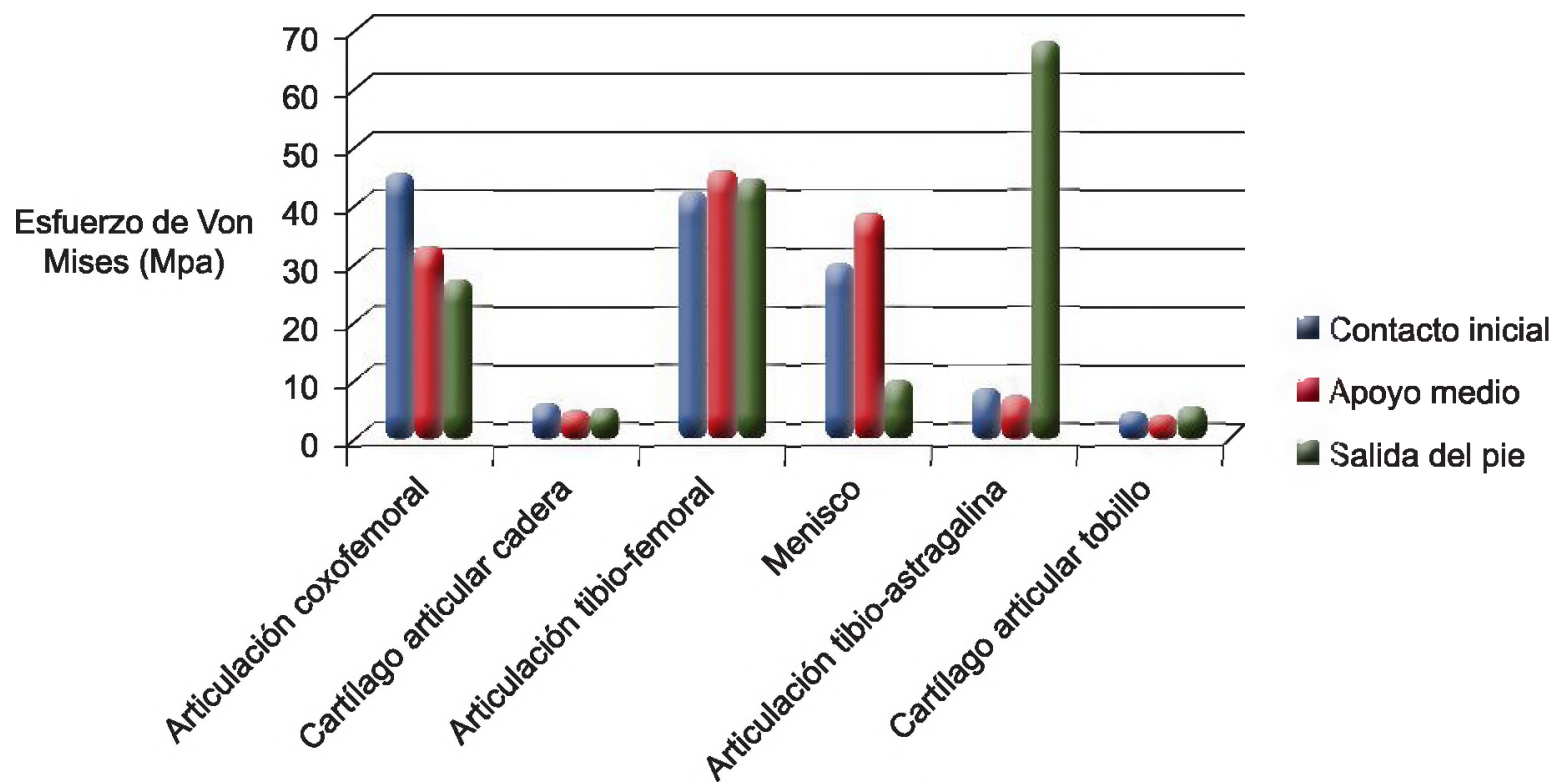

Fuente: los autores 
LÍNEA DE INVESTIGACIÓN: BIOMECÁNICA

La articulación tibioastragalina presento su máximo esfuerzo con el uso de calzado deportivo en la tibia distal con un valor de 60,52 Mpa en fase de salida del pie en la parte posterior de la tibia. El cartílago articular mostro un esfuerzo máximo de 5,1 Mpa.

Los resultados de la simulación con el uso de calzado deportivo se pueden observar en la gráfica 3.

\subsection{Comparación con el uso de plantilla}

Las condiciones del ciclo de trote con el uso de una plantilla presentan una disminución en la fuerza de reacción del suelo. La reducción de los esfuerzos presentes en las articulaciones de la extremidad inferior osciló entre el $7 \%$ y el $10 \%$. La gráfica 4 presenta la comparación de los esfuerzos obtenidos con y sin uso de la plantilla.

\section{Gráfica 3. Resultados obtenidos para uso de calzado deportivo}

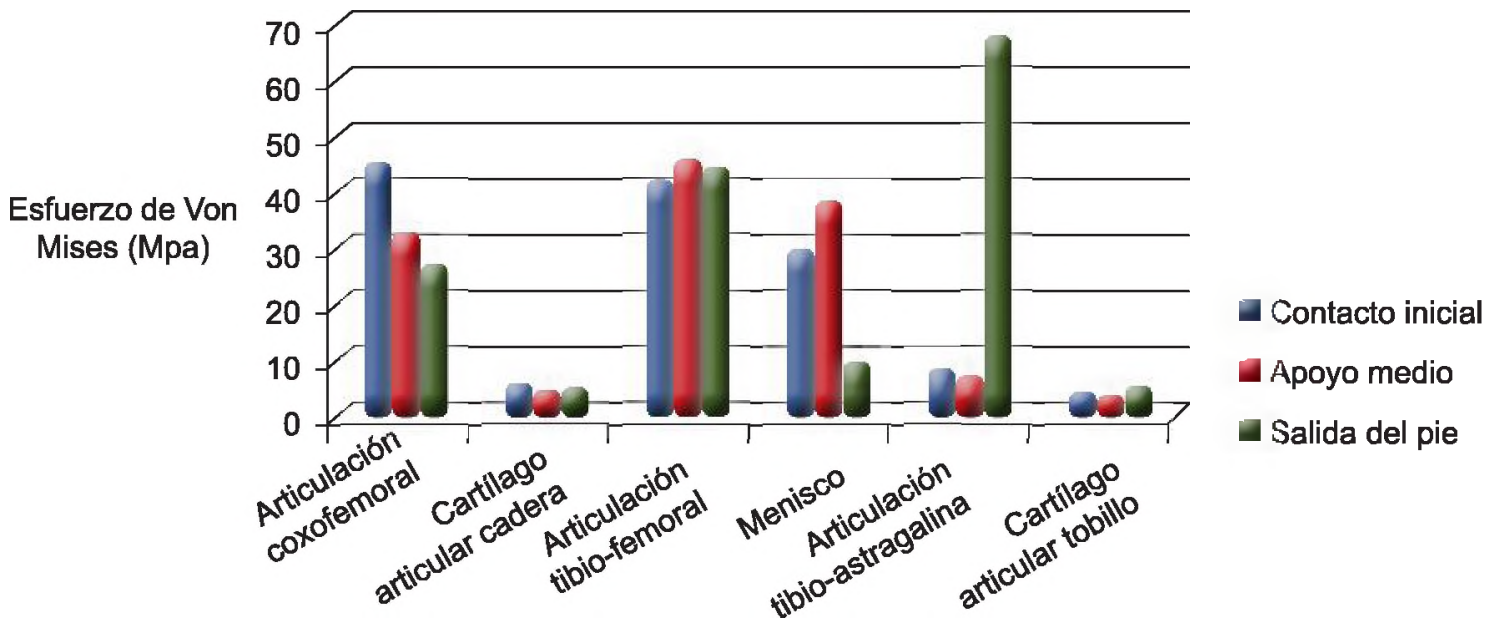

Fuente: los autores

\section{Gráfica 4. Comparación de esfuerzos obtenidos sobre las articulaciones del miembro inferior}

Esfuerzo Von Mises (Mpa)

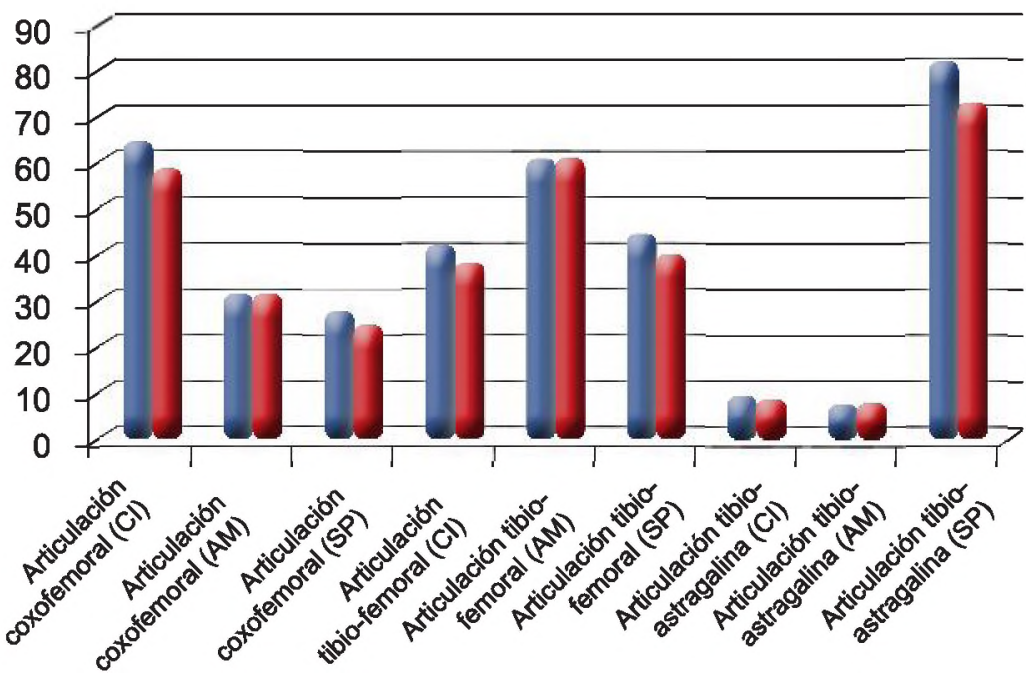

Sin calzado

Con calzado

Fuente: los autores 


\section{DISCUSIÓN Y CONCLUSIONES}

Al aplicar a los modelos geométricos el uso de una plantilla que amortiguara el impacto y el impulso de la extremidad inferior durante la carrera, disminuyó los esfuerzos en un margen de 7 a $10 \%$.

Las fases de contacto inicial en la articulación coxofemoral y salida del pie en la articulación tibioastragalina, se mantienen con un margen de esfuerzos elevados, ratificando que son las fases más críticas en el trote. En las fases de apoyo medio no se presentaron variaciones debido a que durante esta fase la plantilla ya ha amortiguado el impacto. Sin embargo, el apoyo medio presenta su máximo esfuerzo en la articulación tibiofemoral por ser esta la que balancea el cuerpo en el suelo durante el movimiento.

Estos resultados concuerdan con los de anteriores estudios donde el calzado también ha reducido el impacto contra el suelo (O'Leary, Vorpahl, \& Heiderscheit, 2008). No obstante, el porcentaje de producción de la fuerza de impacto observado en el presente estudio, es mayor que los obtenidos en investigaciones previas (Cámara, 2011), donde la marcha con plantillas de material Composite ${ }^{(}$, e Integrale $(\mathcal{C}$, mostraron una reducción de la producción de la fuerza de impacto respecto al ciclo del trote descalza de un $8,58 \%$ y un $14,29 \%$, respectivamente.

La mayor dureza de la suela y la limitación del rango articular del tobillo podrían haber ejercido una influencia en la menor amortiguación.

Otros autores han tomado distintos materiales utilizados en las plantillas, como factores que podrían influir en la amortiguación (Cámara, 2011).
Sin embargo, el menor porcentaje de producción de la fuerza de reacción contra el suelo, con plantilla muestra cuatro, respecto al ciclo del trote descalzo, sugiere que este tipo de plantilla en EVA aporta una protección adicional cuando son implantadas en un calzado convencional.

Como resultado de la investigación, se concluyó que el uso de calzado deportivo, con plantillas fabricadas en materiales como la EVA, generan amortiguaciones, generando una disminución de los esfuerzos del orden del $10 \%$, al disminuir la fuerza de reacción del suelo. Estos resultados son concordantes con los análisis realizados por otros autores. Sin embargo, al trabajar con las tres plantillas que componen el calzado deportivo, podría generar cambios significativos en el porcentaje de amortiguación, ya que significaría tener una suela con mayor espesor, modificando el análisis realizado en la presente investigación.

Por otra parte, los esfuerzos evidenciados en la simulación por elementos finitos para los componentes óseos, no superan el límite de fluencia del hueso compacto (131 MPa) (Beaupied, Lespessilles, \& Bechamou, 2007). No obstante, el manejo de los materiales, como hueso cortical y trabecular puede modificar los resultados obtenidos, debido a que estos dos materiales en conjunto describen mejor el comportamiento del tejido óseo.

Los resultados mostrados por este estudio biomecánico constituyen un aporte a posteriores estudios que en sus objetivos empleen el uso de esfuerzos en las articulaciones de miembro inferior y la interacción con el calzado deportivo convencional.

\section{REFERENCIAS}

Ajay, S., Sherman, M., Reinbolt, J. A., \& Delp, S. L. (2011). "OpemSim: a musculoskeletal modeling and simulation fra- mework for in silico investigations and exchange". Procedia IUTAM, 2, 212-232.

Andreaus, U. A., Colloca, M., \& Toscano, A. (2008). "Mechani- cla Behaviour of a prosthesized human femur: A comparative analysis between walking and stair climbing by using the finite element method". Biophysics and Bioengineering Letters, 1(3). 
LIINEA DE INVESTIGACIÖN: BIOMECÁNICA

Beaupied, H., Lespessilles, E., \& Bechamou, C. L. (2007). "Evaluation of macrostructural bone biomechanics". Joint, Bone, Spine: Revue du Rhumatisme, 74, 233-239.

Cámara, J. (2011). "Comparación de la amortiguación durante la marcha con calzado de montaña respecto a la marcha sin calzado". Revista Universitaria de la Educación Física y el Deporte, 4(4), 37-43.

Chai, H.-M. (2003, 11 05). "Biomechanics of Running". Retrieved from http://www. pt.ntu.edu.tw/hmchai/BM03/ BMsports/Run.htm

De Wit, B., De Clercq, D., \& Aerts, P. (2000). "Biomechanical analysis of the stance phase during barefoot and shod running". Journal of Biomechanics, 33, 269-278.

Dugan, S. A., \& Bhat, K. P. (2005). "Biomechanics and analysis of running gait". Physical and Medicine and Rehabilitation Clinics of North America, $16,602-621$.

Fitzgerald, R., Kaufer, H., \& Malkani, A. (2002). “Ortopedia”.
Montevideo, Uruguay: Editorial Médica Panamericana S.A.

Hammer, S. R., Seth, A., \& Delp, S. L. (2010). "Muscle contributions to propulsion and support during running". Journal fo Biomechanics, 43, 27092716.

Jin-Rae, C., Seung-Bum, P., Sung-Hyun, R., Seung-Ho, K., \& Shi-Bok, L. (2009). "Landing impact analysis of sports shoes using 3-D coupled foot-shoe finite element model". Journal of Mechanical Science and Technology, 23, 2583-2591.

Meardon, S. A., \& Derrick, T. R. (2014). "Effect of step width manipulation on tibial stress during running". Journal of Biomechanics, 47(11), 2738-2744.

Moore, K., Dailey, A., \& Agur, A. (2003"). "Anatomía con orientación clínica”. Montevideo, Uruguay: Editorial Médica Panamericana S.A.

Novacheck, T. F. (1998). "The biomechanics of running". Gait \& Posture, 7(1), 77-95.

O'Leary, K., Vorpahl, K. A., \& Heiderscheit, B. (2008).
"Effect of cushioned insoles on impact forces during running". Journal of the American Podiatric Medical Asssociation, 98, 36- 41.

Palastanga, N., Field, D., \& Roger, S. (2000). "Anatomía y movimiento humano:Estructura y funcionamiento". Barcelona, España: Editorial Paidotribo.

Tortora, G. J., \& Reynolds Grabowski, S. (2012). "Principios de anatomía". México: Editorial Oxford S.A.

Universida Veracruzana. (2015, 05 02). Retrieved from "Sistema esquelético": http:// www.uv.mx/personal/cblazquez/files/2012/01/sistemaoseo.pdf

Yousif, A. E., \& Aziz, M. Y. (2012). "Biomechanical Analysis of the human femur bone during normal". IOSR Journal of Engineering, 2(8), 13-19. 